\title{
Prevalence of Musculoskeletal Pain in Relation to Age, Gender and Anatomical Site Involved
}

\author{
Authors \\ Sujata Hazarika ${ }^{1}$, Miranda Pegu ${ }^{2}$ \\ ${ }^{1}$ Assistant Professor, ${ }^{2}$ Registrar \\ Email: sujatahazarika.sh@gmail.com
}

Dept of Physical Medicine and Rehabilitation, Gauhati Medical College and Hospital, Assam, INDIA

\begin{abstract}
Background: Consistent epidemiologic reports indicate that musculoskeletal pain associated with underlying musculoskeletal disorder is a major medical and economic problem. Pain with or without associated disability is linked with a significant morbidity, loss of productivity and substantial health care expenditures. All age group of male and female population are affected by musculoskeletal pain though the anatomical site involved may vary in different age group.

Aims and Objective: This study was done to know the prevalence of musculoskeletal pain, its gender distribution and the commonly affected age group and also to compare the frequency of the commonly involved anatomical site or region with musculoskeletal pain.

Methodology: This retrospective study was done in a tertiary care hospital. Out of 3215 patients who attended the Physical therapy unit from August to October in the year 2016, 1727 patients who complained of musculoskeletal pain were included in the study.

Results: Prevalence of musculoskeletal pain was 55.2\%, 1039(60\%) were males, 688 (40\%) were female. Most commonly affected age group 31-40years (29.4\%). Low back is the most affected site (54.1\%).

Keywords: Musculoskeletal pain, age, gender, anatomical site.
\end{abstract}

\section{INTRODUCTION}

Pain, as defined by World Health Organisation (WHO) is an unpleasant sensory or emotional experience associated with actual or potential tissue damage or described in terms of such damage. Musculoskeletal pain is pain perceived within a region of the body and believed to arise from the muscles, ligaments, bones or joints in that region .Types of musculoskeletal pain are named according to the region affected eg. Back pain, knee pain etc. Excluded from the definition is pain due to serious local causes as tumors, fractures or infections and systemic or neurological causes. Pain may be mild or severe, acute or chronic, local or widespread. Musculoskeletal pain is the most common presentation of underlying musculoskeletal disorders like osteoarthritis, spondylosis, and rheumatoid arthritis. In the present health scenario more and more patients are reporting with musculoskeletal pain in various body sites as incidence of musculoskeletal disorder is on the rise. Musculoskeletal disorder conditions affect hundreds of millions of people around the world 
and the figure is predicted to rise sharply because of the predicted doubling of the number of people older than the age of fifty by the year 2020 (The Bone and Joint decade, 2000). Increase in life expectancy, life style transition, increasing urbanization, sedentary habits all contribute to the rising incidence of musculo-skeletal disorders. Although not life threatening the patients affected with them constitute a large fraction and they are significantly disabled to engage in productive activities (WHO, 2000). It is reported that pain is the predominant symptom of musculoskeletal disorder by Sharma $\mathrm{R}$ et al ${ }^{(1)}$ Musculoskeletal pain can be regarded as predictor of ensuing disability of the affected part. So due importance should be given to evaluation and treatment of the body part affected with musculoskeletal pain as disability which may follow it will further deteriorate the quality of life. Harkness $\mathrm{EF}$ et $\mathrm{al}^{(2)}$ established in his study that the prevalence of MSP in the year 2005 much higher than in the year 1975 because of increased reporting and increased awareness of the pain symptoms. In a developing country like India, majority of the population engage in hard physical labour for earning means of living and suffer musculoskeletal pain in some phase of their life. Lack of awareness leads to negligence of pain as many regard pain as normal of life which ultimately cause more damage of the site involved. Research regarding awareness and prevention of musculoskeletal pain and evaluation of the underlying musculoskeletal disorder is therefore necessary. Several risk factors are involved in the development of musculoskeletal disorder. Poor work practices, poor fitness, overuse of particular joint or body part, bad posture all contribute to the development of musculoskeletal pain. There have been a number of population based surveys that have estimated the individual disorders of the musculoskeletal system. However few studies have considered relative frequency of musculoskeletal pain at different sites. Special emphasis is therefore given in the present study on the anatomical sites involved with musculoskeletal pain as this knowledge will help in preventing and modifying the risk factors associated with these sites.

\section{AIMS AND OBJECTIVES}

1. To determine the prevalence of musculokeletal pain.

2. To study its demographic profile

3. To determine the body sites or anatomical regions involved.

\section{MATERIALS AND METHODS}

This hospital based retrospective study was conducted in a tertiary care hospital in Northeast part of India. Recorded data of 3215 patients attending physical therapy unit from the period August to October 2016 were retrieved and analysed. The recorded department data included demographic information, referring facility and the presenting features.

\section{Exclusion criteria:}

1) Age less than $10 \mathrm{yrs}$

2) Pain due to systemic and neurological causes

3) Fracture and post operative patients

\section{RESULTS AND OBSERVATION}

Out of 3215 patients, 1488 were excluded by the above mentioned exclusion criteria. The remaining 1727 patients were included in the study and the following results were observed. Gender distribution: In the present study 1039 $(60 \%)$ were male and $688(40 \%)$ were female. The male female ratio was 1.5: 1

Age distribution: The age of patients ranged from 11 to 85 years with maximum belonging to the $31-40$ age group $(29.45 \%)$

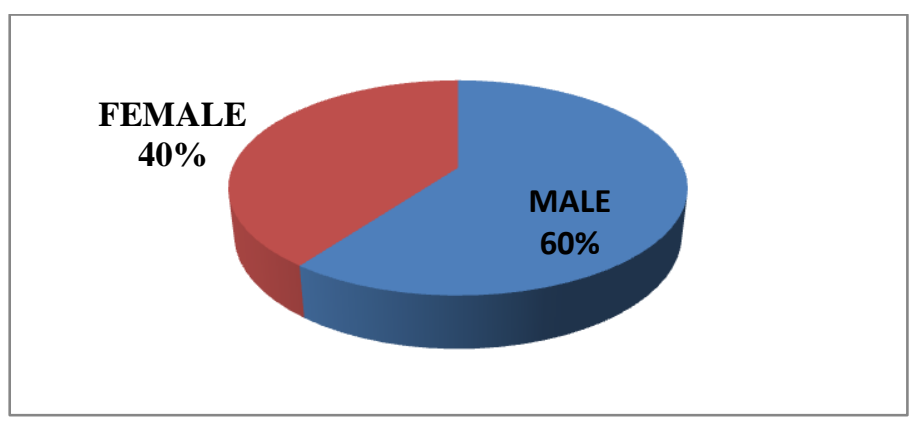

Figure1: Gender distribution 

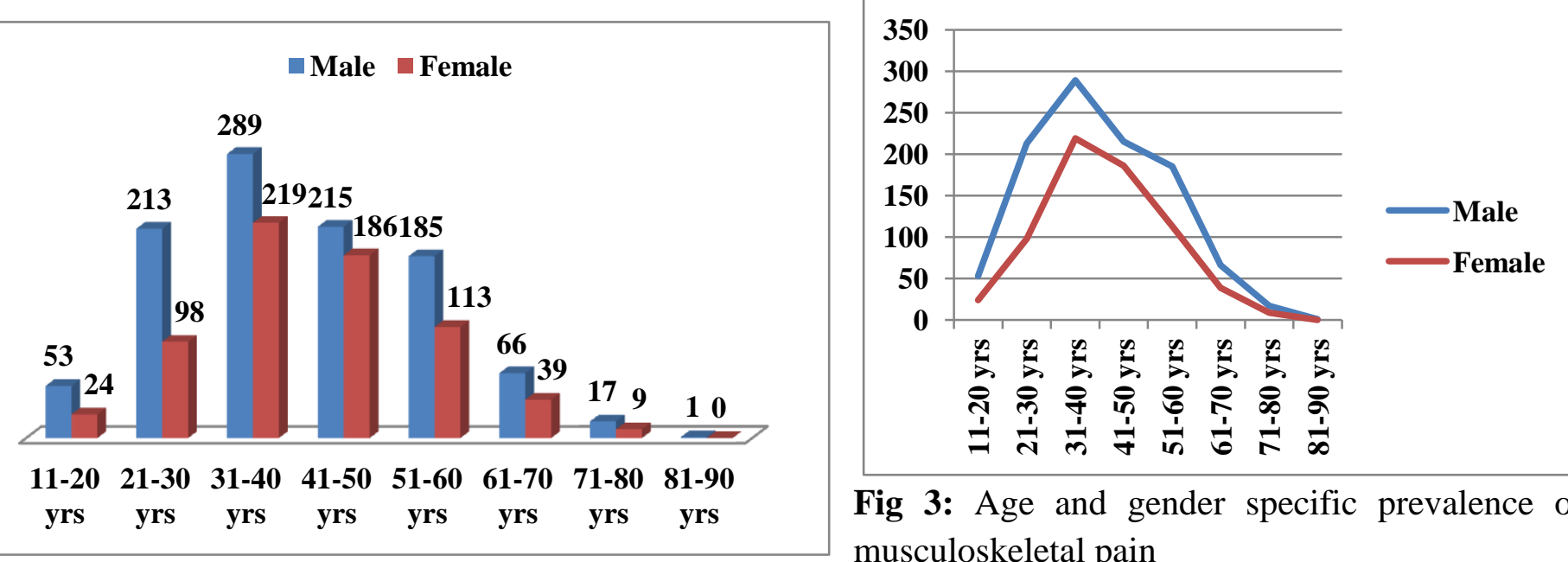

Fig 3: Age and gender specific prevalence of musculoskeletal pain

Fig 2: Age group distribution of the study

Anatomical site involvement: Low back was the population. most commonly involved site $(51.4 \%)$, followed by shoulder (16.7\%), knee (16\%), neck (12.9\%), ankle $(2.3 \%)$, hip $(0.4 \%)$

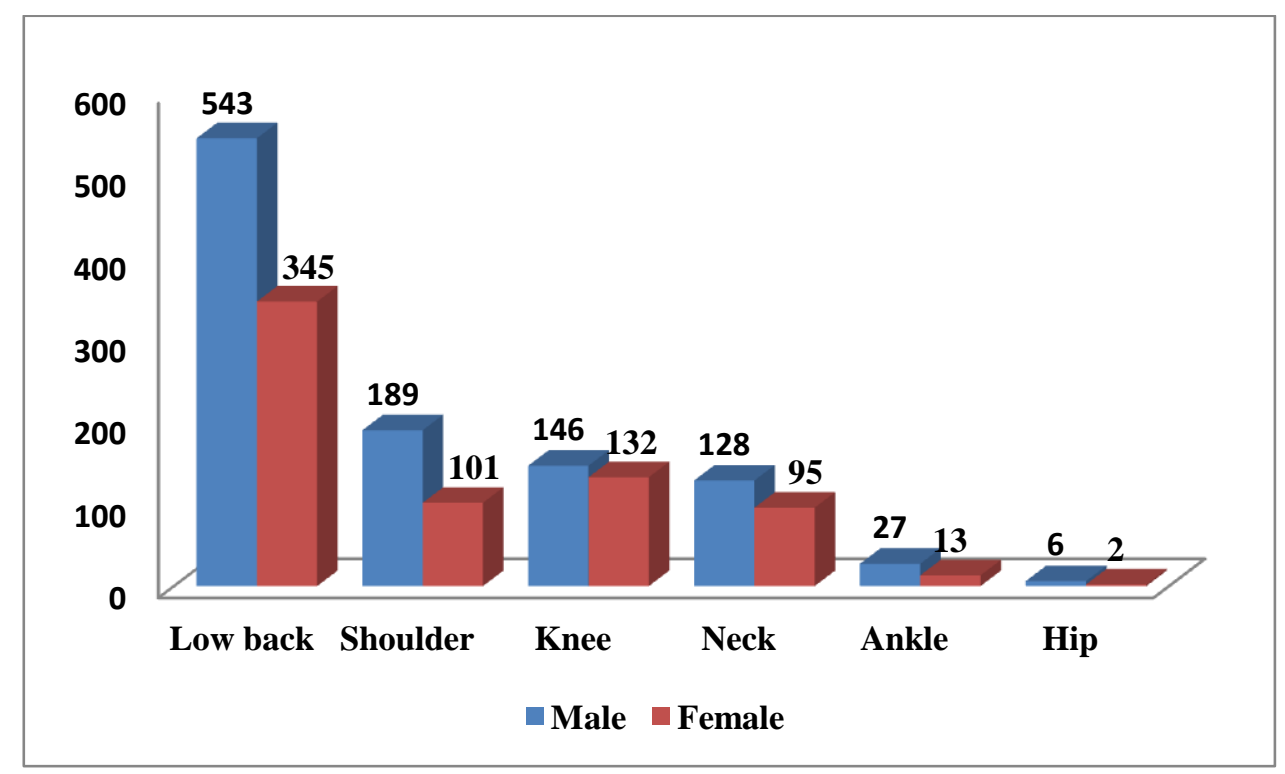

Figure 4: Distribution of patients as per anatomical site involved.

Table 1: Distribution of patients in age group as per anatomical site involved.

\begin{tabular}{|l|c|c|c|c|c|c|c|c|c|}
\hline SITE & TOTAL & $\begin{array}{c}11- \\
20 \mathrm{yrs}\end{array}$ & $\begin{array}{c}21- \\
30 \mathrm{yrs}\end{array}$ & $\begin{array}{c}31- \\
40 \mathrm{yrs}\end{array}$ & $\begin{array}{c}41- \\
50 \mathrm{yrs}\end{array}$ & $\begin{array}{c}51- \\
60 \mathrm{yrs}\end{array}$ & $\begin{array}{c}61- \\
70 \mathrm{yrs}\end{array}$ & $\begin{array}{c}71- \\
80 \mathrm{yrs}\end{array}$ & $\begin{array}{c}81- \\
90 \mathrm{yrs}\end{array}$ \\
\hline Low back & 888 & 30 & 182 & 304 & 191 & 127 & 43 & 11 & 0 \\
\hline Shoulder & 290 & 15 & 35 & 56 & 90 & 65 & 26 & 3 & 0 \\
\hline Knee & 278 & 18 & 37 & 65 & 72 & 58 & 19 & 8 & 1 \\
\hline Neck & 223 & 6 & 49 & 72 & 41 & 39 & 12 & 4 & 0 \\
\hline Ankle & 40 & 8 & 6 & 8 & 6 & 7 & 5 & 0 & 0 \\
\hline Hip & 8 & 0 & 2 & 3 & 1 & 2 & 0 & 0 & 0 \\
\hline
\end{tabular}




\section{DISCUSSION}

Prevalence of musculoskeletal pain in this study was $55.2 \%$. The prevalence of musculoskeletal pain was found to be $25.9 \%$ in the study by Bihari $\mathrm{V}$ et $\mathrm{al}^{(3)}$. High prevalence of musculoskeletal pain in this study may be attributed to association of several risk factors like strenuous and hard physical labour, bad posture, sedentary life, poor workplace condition etc. Both male and female patients were found in the study population but there was male predominance (Figure1) unlike studies by Pingle et al ${ }^{(4)}$, Chopra $\mathrm{A}$ et $\mathrm{al}^{(5)}$ that females are more affected with musculoskeletal pain and disease conditions. Difference of social and occupational roles of men and women, anatomical and physiological differences in the neural systems that transmit or modify pain signals may account for the disparity in the ratio of male female prevalence of musculoskeletal pain. The highest number of male as well as female patients belonged to the age group 31-40 years (29.54\%) (Figure 2). This can be explained by the fact this is the most productive age group in terms of physical and social activity. The prevalence of pain gradually increased with age from 10 to 40 with peak at the 30-40 age group and then gradually declined after age 40 among male and female patients (Figure3).This finding is similar to study by Bihari $\mathrm{V}$ et $\mathrm{al}^{(3)}$ who found that prevalence of musculoskeletal pain increased with age till 50 but later a fall in prevalence. However in a study by Woolf and Pfleger ${ }^{(6)}$ the prevalence of musculoskeletal complaints increased markedly with age. Maximum number of patients presented with low back pain(54.1\%). Shoulder, knee, back of neck, ankle and hip were the other affected sites(Figure4). Urwin $\mathrm{M}$ et al ${ }^{(7)}$ reported common sites of pain as back(23\%), knee(19\%), shoulder $(16 \%)$. Low back pain was more prevalent in the ages from 20 to 60years and maximum prevalence in the 31-40 years age group (34.2\%) (Table 1). Studies have found the incidence of low back pain is highest in the third decade, and overall prevalence increases with age until the 60-65 years age group and then gradually declines ${ }^{(8)}$.

\section{CONCLUSION}

From the present study it can be concluded that prevalence of musculoskeletal pain is on the rising trend. Musculoskeletal pain is therefore a reason of concern for the health care system. It is most common in the age group 31-40 years, males are more affected, and the most commonly involved site is low back. Future research towards age, gender and site specific identification of risk factors for musculoskeletal pain is warranted which will eventually help in the prevention and management of musculoskeletal pain. Public Healthcare Services should focus on educating the masses about postural hygiene, physical exercise, to avoid sedentary lifestyle habits to prevent painful musculoskeletal conditions.

\section{REFERENCES}

1. Sharma R, editor: Epidemiology of Musculoskeletal conditions in India. New Delhi,India: Indian Council of Medical Research(ICMR);2012

2. Harkness EF, Macfarlane GJ, Silman AJ, McBeth J. Is musculoskeletal pain more common now than 40 years ago? Two population-based cross sectional studies. Rheumatology (Oxford).2005 Jul;44 (7): 890-5

3. Bihari V,Kesavachandran C,PangteyB S,Srivastava AK,Mathur N. Musculoskeletal pain and its associated risk factors in residents of national capital region. Indian J Occup Environ Med 2011;15:5963

4. Pingle AS, Pabdit DD. A cross sectional study of Rheumatic Musculoskeletal disorders in an urban slum population. Indian J Community Med. 2006; 31:244-5

5. Chopra A, Patil V,RelwaniJ,Tandale HS. Prevalence of rheumatic diseases in a rural J Billempelly population in Western India, J Assoc Physicians India.2001:49:240-6h 
6. Woolf AD ,Plfeger B. Burden of major musculoskeletal conditions. Bull World Health Organ. 2003;81:646-56

7. Urwin $\mathrm{M}$, Symmons $\mathrm{D}$, Allison $\mathrm{T}$, Brammah T, Busby H, Roxy M, et al. Estimating the burden of musculoskeletal disorders in the community: The comparative prevalence of symptoms at different anatomical sites and the relation to social deprivation. Ann Rheum Dis. 1998; 57:649-55

8. Best Pract Res Clin Rheumatol.2010 Dec;24(6):769-81

doi:10.1016/j.berh.2010.10.002 